Pacific

Journal of

Mathematics

RELATIVE TOPOLOGY OF REAL ALGEBRAIC VARIETIES IN THEIR COMPLEXIFICATIONS

YILDIRAY OZAN 


\title{
RELATIVE TOPOLOGY OF REAL ALGEBRAIC VARIETIES IN THEIR COMPLEXIFICATIONS
}

\author{
YILDIRAY OZAN
}

\begin{abstract}
We investigate, for a given smooth closed manifold $M$, the existence of an algebraic model $X$ for $M$ (i.e., a nonsingular real algebraic variety diffeomorphic to $M$ ) such that some nonsingular projective complexification $i: X \rightarrow X_{\mathbb{C}}$ of $X$ admits a retraction $r: X_{\mathbb{C}} \rightarrow X$. If such an $X$ exists, we show that $M$ must be formal in the sense of Sullivan's minimal models, and that all rational Massey products on $M$ are trivial.

We also study the homomorphism on cohomology induced by $i$ for algebraic models $X$ of $M$. Using étale cohomology, we see that $\bmod p$ Steenrod powers give an obstruction for the induced map on cohomology, $i^{*}: H^{k}\left(X_{\mathbb{C}}, \mathbb{Z}_{p}\right) \rightarrow H^{k}\left(X, \mathbb{Z}_{p}\right)$, to be onto, if we require that $X$ is defined over rational numbers.
\end{abstract}

\section{Introduction}

Let $M$ be a closed smooth manifold. In [T] Tognoli, generalizing the results of Seifert $[\mathbf{S}]$ and Nash $[\mathbf{N}]$, proved that there is a nonsingular real algebraic variety $X$ diffeomorphic to $M$, which we call an algebraic model of $M$. Later Akbulut and King improved Tognoli's result, proving that if $M \subseteq \mathbb{R}^{N}$ then $M$ can be isotoped to a nonsingular real algebraic subvariety of $\mathbb{R}^{N} \times \mathbb{R}$ [AK3, AK4].

In 1978 Kulkarni considered the following problem in $[\mathbf{K u}]$ : Is there an algebraic model $X$ of $M$ such that the inclusion $i: X \rightarrow X_{\mathbb{C}}^{0}$ of $X$ into some quasiprojective complexification $X_{\mathbb{C}}^{0}$ is a homotopy equivalence? He calls such $X_{\mathbb{C}}^{0}$ a minimal complexification of $M$. Using mixed Hodge structures he showed that if $M$ has a minimal complexification the Euler characteristic of $M$ is nonnegative. He also showed that any homogeneous space $G / H$, where $G$ is a compact Lie group, admits a canonical minimal complexification.

Since any nonsingular complex projective variety has a fundamental class, by dimension reasons the inclusion of $X$ into any nonsingular projective complexification, $i: X \rightarrow X_{\mathbb{C}}$, is never a homotopy equivalence. Instead one can consider the following problem: Is there an algebraic model $X$ of $M$ with a smooth projective complexification, $i: X \rightarrow X_{\mathbb{C}}$, that admits a retraction $r: X_{\mathbb{C}} \rightarrow X$ ? 
If $M$ is $S^{1}$ or a closed orientable surface, $M$ has an algebraic model $X$ with a smooth projective complexification that retracts onto $X$. In dimensions larger than two it turns out that, as in the case of Kulkarni's result, there are topological obstructions to the existence of such $X$ in terms of Massey products and Sullivan's rational minimal models:

Theorem 1.1. Let $M$ be a closed smooth manifold admitting an algebraic model $X$ with a smooth projective complexification that retracts onto $X$. Then, $M$ is formal. In particular, all Massey products in $M$ are trivial.

This is an immediate consequence of Theorems 2.1 and 2.3. The following corollary of Theorem 1.1 will be proved in Section 2:

Corollary 1.2. Let $N$ be the total space of an $S^{1}$-bundle over an orientable closed surface $F$ of positive genus. Then $N$ has an algebraic model $X$ with a projective complexification that retracts onto $X$ if and only if the $S^{1}$-bundle is trivial; that is, $N=F \times S^{1}$.

In Section 3, we will see that the manifold $N$ in this corollary has an algebraic model $X$ such that $i^{*}: H^{*}\left(X_{\mathbb{C}}, \mathbb{Z}\right) \rightarrow H^{*}(X, \mathbb{Z})$ is surjective for any projective complexification $X_{\mathbb{C}}$ (Corollary 3.3 ). However, by the result above, none of these complexifications retract onto $X$.

On the positive side we have the following result:

Proposition 1.3. Let $Z$ be a smooth complex projective variety. Regarded as smooth manifolds, both $Z$ and $Z \times S^{1}$ admit algebraic models with smooth projective complexifications that retract onto them.

Remark 1.4. Let $F_{g}$ be a Riemann surface of genus $g$. The algebraic surface $\left(F_{g} \times \mathbb{C P}^{1}\right) \curvearrowleft n \overline{\mathbb{C P}^{2}}$, the blowup of $F_{g} \times \mathbb{C P}^{1}$ at $n$ points, has Euler characteristic $4(1-g)+n$, and this can be any integer if $n$ and $g$ are chosen appropriately. Hence, unlike Kulkarni's result, in Theorem 1.1 there is no restriction on the Euler characteristic.

In the next section we review Sullivan's theory of minimal models, formality and Massey products. At the end of that section we construct manifolds with nonnegative Euler characteristic that do not admit minimal complexifications in the sense of Kulkarni's result. Hence, the condition in Kulkarni's theorem that $\chi(M) \geq 0$ is necessary but not sufficient.

In Section 3, we first review some basic material in real algebraic geometry, needed to prove Proposition 1.3. Then we study the homomorphism on cohomology, induced by $i: X \rightarrow X_{\mathbb{C}}$, for algebraic models $X$ of a smooth closed manifold $M$. In particular, using étale cohomology we see that $\bmod p$ Steenrod powers give an obstruction for the induced map on cohomology, $i^{*}: H^{k}\left(X_{\mathbb{C}}, \mathbb{Z}_{p}\right) \rightarrow H^{k}\left(X, \mathbb{Z}_{p}\right)$, to be onto, whenever $X$ is defined over $\mathbb{Q}$. 


\section{Minimal models, formality and Massey products}

In this section we mainly follow [GM] and [DGMS]. A differential graded algebra is an algebra $\mathcal{A}=\bigoplus_{k>0} \mathcal{A}^{k}$ with a differential $d: \mathcal{A} \rightarrow \mathcal{A}$ of degree +1 , such that $\mathcal{A}$ is graded commutative (i.e., $x y=(-1)^{\operatorname{deg} x \operatorname{deg} y} y x$ ) and $d$ is a derivation with $d^{2}=0$. We will denote its cohomology by $H^{*}(\mathcal{A})$. Note that $H^{*}(\mathcal{A})$ together with the zero differential, $d=0$, is again a differential graded algebra. In this work, $\mathcal{A}^{0}$ denotes the ground field, which will be either $\mathbb{Q}$ or $\mathbb{R}$. Each $H^{k}(\mathcal{A})$ is assumed to be finite-dimensional.

If $H^{0}(\mathcal{A})=\mathcal{A}^{0}$ then $\mathcal{A}$ is called connected, and if also $H^{1}(\mathcal{A})=0$ then $\mathcal{A}$ is called 1-connected.

A minimal differential graded algebra $\mathcal{M}$ is a differential graded algebra such that:

(1) $\mathcal{M}$ is free as a graded commutative algebra; i.e., it is a tensor product of polynomial algebras on generators of even degrees and exterior algebras on generators of odd degrees.

(2) $d$ is decomposable; i.e., $d(\mathcal{M}) \subseteq \mathcal{M}^{+} \wedge \mathcal{M}^{+}$, where $\mathcal{M}^{+}=\bigcup_{i>0} \mathcal{M}_{i}$.

A minimal model for a differential graded algebra $\mathcal{A}$ is a degree-zero homomorphism $\phi: \mathcal{A} \rightarrow \mathcal{M}$ to a minimal algebra $\mathcal{M}$, inducing an isomorphism on cohomology.

It is well-known that any connected differential algebra $\mathcal{A}$ having finitedimensional cohomology in each degree has a minimal model $\phi: \mathcal{A} \rightarrow \mathcal{M}$. Moreover, $\mathcal{M}$ is unique up to isomorphism.

Let $K$ be a simplical complex and $E^{*}(K)$ the rational de Rham complex of $K$, that is, the complex of $\mathbb{Q}$-polynomial forms on $K$. For a smooth manifold $M$, let $\mathcal{E}^{*}(M)$ denote the differential algebra of smooth differential forms on $M$. Minimal models of these two algebras are related as follows: if $K$ is a $C^{1}$-triangulation of $M$, the minimal model of $\mathcal{E}^{*}(M)$ is isomorphic to the minimal model of $E^{*}(K)$ tensored with $\mathbb{R}$.

For a simply connected finite $C W$-complex $K$, the rational homotopy of $K$ can be read from its rational minimal model. Namely, $\pi_{i}(K) \otimes \mathbb{Q} \cong$ $I^{i}(K)$, where $I^{i}(K)$ denotes the vector space of irreducibles of degree $i$ in $\mathcal{M}\left(E^{*}(K)\right)$.

A differential graded algebra $\mathcal{A}$ is called formal if there is a map $\psi: \mathcal{A} \rightarrow$ $H^{*}(\mathcal{A})$ of degree zero and inducing isomorphism on cohomology, the latter being endowed with the zero differential map. This is equivalent to saying that $\mathcal{A}$ and $H^{*}(\mathcal{A})$ have isomorphic minimal models.

A smooth manifold $M$ is called formal if $\mathcal{E}^{*}(M)$ is formal. Some examples of formal manifolds are wedges of spheres, compact connected Lie groups, Eilenberg-Mac Lane spaces $K(\pi, n), n>1$, Riemannian symmetric spaces and compact Kähler manifolds.

Next we give an obstruction against $M$ being formal, in terms of Massey products. Let $X$ be a space and let $a, b, c \in H^{*}(X, R)$ classes of degree $p, q, r$ 
respectively, such that $a \cup b=0=b \cup c$, where $R$ is any commutative ring with unity. Choose cochain representatives $\alpha, \beta, \gamma$ for $a, b, c$ respectively. Also let $\mu$ and $\tau$ be cochains such that $d \mu=\alpha \wedge \beta$ and $d \tau=\beta \wedge \gamma$. Then

$$
\mu \wedge \gamma-(-1)^{p} \alpha \wedge \tau
$$

is a closed form, called a triple Massey product of $a, b, c$. It passes to a well-defined class in the quotient group

$$
H^{p+q+r-1}(X, R) /\left(a \cup H^{q+r-1}(X, R)+c \cup H^{p+q-1}(X, R)\right),
$$

called the Massey triple product of the classes $a, b, c$ and denoted by $\langle a, b, c\rangle$. We will also use the same notation $\langle a, b, c\rangle$ to denote the coset

$$
\mu \wedge \gamma-(-1)^{p} \alpha \wedge \tau+\left(a \cup H^{q+r-1}(X, R)+c \cup H^{p+q-1}(X, R)\right) ;
$$

i.e., the set of all Massey triple products of the classes $a, b, c$. There are also higher Massey products, $\left\langle a_{1}, a_{2}, \ldots, a_{k}\right\rangle$, which are defined if all the lower Massey products formed from the elements $a_{1}, a_{2}, \ldots, a_{k}$ are zero.

Massey products are functorial in the sense that if $f: X \rightarrow Y$ is a continuous map then

$$
f^{*}\left(\left\langle a_{1}, a_{2}, \ldots, a_{k}\right\rangle\right) \subseteq\left\langle f^{*}\left(a_{1}\right), f^{*}\left(a_{2}\right), \ldots, f^{*}\left(a_{k}\right)\right\rangle .
$$

In particular, if $f^{*}$ is an isomorphism on cohomology it preserves Massey products.

Indeed, Massey products can be defined in any differential graded algebra $(\mathcal{A}, d)$; all higher Massey products are zero if $d=0$. Hence, on a smooth formal manifold $M$ all higher rational Massey products are 0 . In particular:

Theorem 2.1 ([DGMS]). Any compact Kähler manifold $M$ is formal. In particular, all higher rational Massey products in $M$ are zero.

Any minimal model $\mathcal{M}$ is isomorphic to

$$
P\left[V_{2} \oplus V_{4} \oplus \cdots\right] \otimes \bigwedge\left[V_{1} \oplus V_{3} \oplus \cdots\right],
$$

where each $V_{i}$ is a vector space that contains elements of degree $i$ only and $P\left[V_{2} \oplus V_{4} \oplus \cdots\right]$ is the polynomial algebra part and $\bigwedge\left[V_{1} \oplus V_{3} \oplus \cdots\right]$ the exterior algebra part of $\mathcal{M}$.

Let $C_{i}$ denote the subspace of closed elements in $V_{i}$. The theorem below shows that $\mathcal{M}$ is formal if and only if all Massey products are zero in a uniform way:

Theorem 2.2 ([DGMS $]) \cdot \mathcal{M}$ is formal if and only if there is in each $V_{i}$ a complement $N_{i}$ to $C_{i}$, such that any closed form a in the ideal $I\left(\oplus N_{i}\right)$ is exact. Choosing such an $N_{i}$ is equivalent to choosing a $\psi: \mathcal{M} \rightarrow H^{*}(\mathcal{M})$ that induces an isomorphism on cohomology.

The key observation of this section is: 
Theorem 2.3. Let $M$ be closed smooth manifold such that there exists a retraction $r: N \rightarrow M$, where $N$ is a closed smooth formal manifold. Then $M$ is formal. In particular, all higher rational Massey products in $M$ are zero.

\section{Remark 2.4.}

(1) Theorem 1.1 follows from Theorems 2.1 and 2.3.

(2) It follows from the preceding theorem that the product $M_{1} \times M_{2}$ of two closed manifolds is formal if and only if $M_{1}$ and $M_{2}$ are formal.

(3) As stated before, any compact Lie group is formal. On the other hand, we know that any compact Lie group has the structure of a unique real linear algebraic group $[\mathbf{D M}]$ that and $G$, equipped with this real algebraic structure, satisfies the following: for any smooth projective complexification $i: G \rightarrow G_{\mathbb{C}}$, the induced map on cohomology $i^{*}: H^{i}\left(G_{\mathbb{C}}, \mathbb{Q}\right) \rightarrow H^{i}(G, \mathbb{Q})$ is trivial for $i>0$ (see $[\mathbf{O} 1]$ ). Hence, for this canonical algebraic structure there is no smooth projective complexification of $i: G \rightarrow G_{\mathbb{C}}$ that retracts onto $G$. On the contrary, in Kulkarni's result mentioned in the introduction the affine complexification of $G$ is homotopy equivalent to $G$ via the inclusion map.

Proof of Theorem 2.3. Let $\mathcal{M}$ and $\mathcal{N}$ denote the minimal models of $M$ and $N$ respectively. We know that

$$
\mathcal{N} \simeq P\left[V_{2} \oplus V_{4} \oplus \cdots\right] \otimes \bigwedge\left[V_{1} \oplus V_{3} \oplus \cdots\right]
$$

and

$$
\mathcal{M} \simeq P\left[V_{2}^{\prime} \oplus V_{4}^{\prime} \oplus \cdots\right] \otimes \bigwedge\left[V_{1}^{\prime} \oplus V_{3}^{\prime} \oplus \cdots\right]
$$

where $V_{i}$ and $V_{i}^{\prime}$ are the vector subspaces that contain the elements of degree $i$ only. The maps $i: M \rightarrow N$ and $r: N \rightarrow M$ induce homomorphisms $i^{*}: \mathcal{M} \rightarrow \mathcal{N}$ and $r^{*}: \mathcal{N} \rightarrow \mathcal{M}$ such that $i^{*} \circ r^{*}=\left.\mathrm{id}\right|_{\mathcal{M}}$.

Let $C_{i}$ and $C_{i}^{\prime}$ denote the subspaces of closed elements in $V_{i}$ and $V_{i}^{\prime}$. Since $N$ is formal, by Theorem 2.2 there is in each $V_{i}$ a complement $N_{i}$ to $C_{i}$ such that any closed form $a$ in the ideal $I\left(\oplus N_{i}\right)$ is exact. Then $V_{i}^{\prime}=\left(V_{i}^{\prime} \cap C_{i}\right) \oplus$ $\left(V_{i}^{\prime} \cap N_{i}\right)$, where $\left(V_{i}^{\prime} \cap C_{i}\right)$ is clearly $C_{i}^{\prime}$. Hence, again by Theorem 2.2 , it suffices to show that any closed element $a^{\prime}$ in the ideal $I\left(\oplus N_{i}^{\prime}\right)$ is exact, where $N_{i}^{\prime}=V_{i}^{\prime} \cap N_{i}$.

Let $a^{\prime}$ be a closed element in the ideal $I\left(\oplus N_{i}^{\prime}\right)$. Then $a=r^{*}\left(a^{\prime}\right)$ is a closed element in $I\left(\oplus N_{i}\right)$ and thus $a=d(b)$ for some $b \in \mathcal{N}$. Now, $a^{\prime}=i^{*}(d(b))=d\left(i^{*}(b)\right)$ finishes the proof.

Although the proof below is well-known to experts, we will reproduce it here for the sake of completeness.

Proof of Corollary 1.2. Let $N$ be the total space of an $S^{1}$-bundle over an orientable closed surface $F$ of positive genus. Suppose that the first Chern 
class of the $S^{1}$-bundle is nonzero. The Gysin sequence associated to the $S^{1}$ bundle implies that $H^{1}(F, \mathbb{R})$ maps isomorphicaly onto $H^{1}(N, \mathbb{R})$ and $H^{2}(F, \mathbb{R})$ maps to zero in $H^{2}(N, \mathbb{R})$. In particular, we may identify $H^{1}(F, \mathbb{R})$ with $H^{1}(N, \mathbb{R})$. Let $a, b \in H^{1}(F, \mathbb{R})$ such that $c_{1}(N)=a \cup b$. Then by Chern-Weil theory there is a connection on the $S^{1}$-bundle whose associated 1-form $\eta$ satisfies $d(\eta)=c_{1}(N)=a \cup b$. Moreover,

$$
(\eta \cup a \cup b)([N])=(\eta \cup d(\eta))([N])=c_{1}(N)([F]) \neq 0 .
$$

Hence, the Massey product $\langle a, a, b\rangle$, which is represented by $\eta \cup a$, is not zero. In particular, $N$ is not formal.

The 'if' part of the Corollary follows from Proposition 1.3.

Example 2.5. One can find simply connected smooth manifolds containing nonzero Massey products as follows: let $K$ be a simply connected finite $C W$ complex with nontrivial Massey products. For example, let $K$ be a finite $C W$-approximation of the free loop space $\Lambda S^{2 k}, k>1$, which supports a nontrivial Massey product (see $[\mathbf{S V P}]$ ). Embed $K$ into some Euclidean space, $\mathbb{R}^{N}$, and let $M$ be the double of a tubular neighborhood $\nu(K)$ in $\mathbb{R}^{N}$. Since $M$ retracts onto $K$, by Theorem 2.3 $M$ contains nonzero Massey products.

Example 2.6. For finitely presentable groups there is another notion of formality, called 1-formality. The fundamental group of any formal space is 1-formal. The group

$$
\Gamma=\langle x, y, z, t \mid[x, y][z, t],[[[[y, x], x], x], y]\rangle
$$

is not 1-formal; see [ABCKT, Proposition 3.20 and Example 3.22, pp. 3238]. Therefore, if $M$ is any smooth manifold with fundamental group $\Gamma-$ such an $M$ exists in dimensions at least 4 (see the remark below) $-M$ is not formal but the Massey triple products of elements in $H^{1}(M, \mathbb{Q})$ are all zero. In particular:

Corollary 2.7. If $M$ is a smooth manifold with fundamental group $\Gamma$ as above, $M$ does not admit an algebraic model $X$ with a smooth projective complexification that retracts onto $X$.

Remark 2.8. By a result of Morgan, the group $\Gamma$ of Example 2.6 cannot be the fundamental group of a smooth quasiprojective complex variety (see Corollary 3.53 and the subsequent paragraph in [ABCKT, p. 463]). Construct a 4-manifold $N$ by taking the connected sum of four copies of $S^{3} \times S^{1}$ and gluing two 2-handles along smooth embedded loops representing the relations of $\Gamma$. Then $N$ has $\Gamma$ as its fundamental group and its Euler characteristic is -2 . So, if $n \geq 2$, the connected sum $M=N \curvearrowleft n \mathbb{C P}^{2}$ has nonnegative Euler characteristic $n-2$ and fundamental group $\Gamma$. Hence, the condition in Kulkarni's theorem that $\chi(M) \geq 0$ is necessary but not sufficient. 


\section{Algebraic models}

All real algebraic varieties considered here are compact and nonsingular. It is well-known that real projective varieties are affine (Proposition 2.4.1 of [AK2] or Theorem 3.4.4 of [BCR]). Compact affine real algebraic varieties are projective (Corollary 2.5.14 of [AK2]), so we will not distinguish between real compact affine varieties and real projective varieties.

For real algebraic varieties $X \subseteq \mathbb{R}^{r}$ and $Y \subseteq \mathbb{R}^{s}$ a map $F: X \rightarrow Y$ is said to be entire rational if there exist $f_{i}, g_{i} \in \mathbb{R}\left[x_{1}, \ldots, x_{r}\right], i=1, \ldots, s$, such that each $g_{i}$ vanishes nowhere on $X$ and $F=\left(f_{1} / g_{1}, \ldots, f_{s} / g_{s}\right)$. We say $X$ and $Y$ are isomorphic if there are entire rational maps $F: X \rightarrow Y$, $G: Y \rightarrow X$ such that $F \circ G=\mathrm{id}_{Y}$ and $G \circ F=\mathrm{id}_{X}$. We regard isomorphic algebraic varieties as being the same. A complexification $X_{\mathbb{C}} \subseteq \mathbb{C P}^{N}$ of $X$ will mean that $X$ is embedded into some projective space $\mathbb{R P}^{\bar{N}}$ and $X_{\mathbb{C}} \subseteq$ $\mathbb{C P}^{N}$ is the complexification of the pair $X \subseteq \mathbb{R P}^{N}$. We also require the complexification to be nonsingular (blow up $X_{\mathbb{C}}$ along smooth centers away from $X$ defined over reals if necessary, $[\mathbf{H i}, \mathbf{B M}]$ ). We refer the reader for the basic definitions and facts about real algebraic geometry to $[\mathbf{A K 2}, \mathbf{B C R}]$.

Suppose that $R$ is a commutative ring with unity and $X$ is $R$-orientable. Let $K H_{k}(X, R)$ denote the kernel of the induced map

$$
i_{*}: H_{k}(X, R) \rightarrow H_{k}\left(X_{\mathbb{C}}, R\right)
$$

on homology. Then $K H_{k}(X, R)$ is independent of the complexification $X \subseteq$ $X_{\mathbb{C}}$ and thus an (entire rational) isomorphism invariant of $X$. Similarly, the image of the homomorphism

$$
i^{*}: H^{k}\left(X_{\mathbb{C}}, R\right) \rightarrow H^{k}(X, R),
$$

denoted by $\operatorname{Im} H^{k}(X, R)$, is also an isomorphism invariant of $X$, [O2]. In [BK2], Bochnak and Kucharz also studied $K H_{k}(X, R)$ independently.

\section{Example 3.1.}

a) If $Z$ is a compact nonsingular complex algebraic variety, we can view $Z$ as a real algebraic variety, which we denote by $Z_{\mathbb{R}}$. This is just the fixed-point set of the antiholomorphic involution $\sigma: Z \times \bar{Z} \rightarrow$ $Z \times \bar{Z}$ given by $\sigma(x, y)=(\bar{y}, \bar{x})$, where $\bar{Z}$ is the complex conjugate of $Z$. It is well-known that there is a complex algebraic subvariety $W$ of some projective space $\mathbb{C P}^{N}$ defined by real polynomials which is biregularly isomorphic to $Z \times \bar{Z}$. Moreover, the real part $W \cap \mathbb{R P}^{N}$ is isomorphic to $Z_{\mathbb{R}}$. However, any projective real algebraic variety is affine (Proposition 3.4.4 in [BCR]) and hence $Z_{\mathbb{R}}$ can be viewed as an affine real algebraic variety (see Sections 1 and 2 of $[\mathbf{H u}]$ ). Now clearly, there is a retraction $W=\left(Z_{\mathbb{R}}\right)_{\mathbb{C}} \rightarrow Z_{\mathbb{R}}$, and therefore $\operatorname{Im} H^{*}\left(Z_{\mathbb{R}}, R\right)=$ $H^{*}\left(Z_{\mathbb{R}}, R\right)$. In particular, for $\mathbb{C P}^{n}$, regarded as a real algebraic variety, we have $\operatorname{Im} H^{*}\left(\mathbb{C P}^{n}, R\right)=H^{*}\left(\mathbb{C P}^{n}, R\right)$; see $[\mathbf{O 2}]$. 
b) The quaternion projective space $\mathbb{H}^{n}$ has also a canonical real algebraic structure. The canonical quaternion line bundle $\xi$ over $\mathbb{H} \mathbb{P}^{n}$ is strongly algebraic $[\mathbf{B B K}]$ and thus $p_{1}(\xi) \in \operatorname{Im} H^{4}\left(\mathbb{H P}^{n}, R\right)[\mathbf{O 2}$, AK5]. In particular, $\operatorname{Im} H^{*}\left(\mathbb{H}_{\mathbb{P}^{n}}, R\right)=H^{*}\left(\mathbb{H} \mathbb{P}^{n}, R\right)$.

c) Let $X$ be any nonsingular real algebraic curve diffeomorphic to $S^{1}$ such that $X_{\mathbb{C}}-X$ is connected. So, topologically, $X_{\mathbb{C}}$ is an orientable closed surface and $X$ is a nonseparating simple closed curve on it. Again there is a retraction of $X_{\mathbb{C}}$ onto $X$ (and therefore $\operatorname{Im} H^{*}(X, R)=H^{*}(X, R)$ ).

Since $X$ is a nonseparating simple closed curve on $X_{\mathbb{C}}$, its homotopy class, say $\alpha$, is an element of some generating set for the fundamental group $\pi_{1}\left(X_{\mathbb{C}}\right)$. Also, up to homotopy, a retraction $X_{\mathbb{C}} \rightarrow X$ is completely determined by the induced surjective homomorphism $\pi_{1}\left(X_{\mathbb{C}}\right) \rightarrow \pi_{1}(X) \simeq\langle\alpha\rangle$ sending $\alpha$ to itself. In particular, there are many homotopically different retractions.

Proof of Proposition 1.3. The proof follows from parts (a) and (c) of the preceding example.

Theorem 3.2. Let $M$ be closed smooth manifold. Then $M$ has an algebraic model $X$ such that $\operatorname{Im} H^{*}(X, \mathbb{Z})$ contains $H^{k}(X, \mathbb{Z})$ for $k=1,2$, 4. In particular, if the cohomology ring $H^{*}(X, \mathbb{Z})$ is generated by elements of degree 1,2 and 4 then $\operatorname{Im} H^{*}(X, \mathbb{Z})=H^{*}(X, \mathbb{Z})$.

In case of rational coefficients, $M$ has an algebraic model $X$ such that $\operatorname{Im} H^{*}(X, \mathbb{Q})$ contains $H^{\text {even }}(X, \mathbb{Q})$ and $H^{1}(X, \mathbb{Q})$.

As an immediate consequence we get:

Corollary 3.3. Any closed smooth three-manifold with positive first Betti number has an algebraic model $X$ such that $\operatorname{Im} H^{*}(X, \mathbb{Z})=H^{*}(X, \mathbb{Z})$.

Proof of Theorem 3.2. Choose generating sets $a_{1}, \ldots, a_{n_{1}}, b_{1}, \ldots, b_{n_{2}}$ and $c_{1}, \ldots, c_{n_{4}}$ for the $\mathbb{Z}$-modules $H^{1}(M, \mathbb{Z}), H^{2}(M, \mathbb{Z})$ and $H^{4}(M, \mathbb{Z})$. We can regard each $a_{i}, b_{i}$ and $c_{i}$ as a smooth map $a_{i}: M \rightarrow K(\mathbb{Z}, 1)=S^{1}$, $b_{i}: M \rightarrow K(\mathbb{Z}, 2)=\mathbb{C P}^{N}$ and $c_{i}: M \rightarrow K(\mathbb{Z}, 4)=\mathbb{H} \mathbb{P}^{N}$ for $N$ large enough $(2 N>\operatorname{dim} M$ would suffice).

It is well-known that the Grassmann varieties, in particular $\mathbb{C P}^{N}$ and $\mathbb{H} \mathbb{P}^{N}$, have canonical real algebraic structures that have totally algebraic homology [AK1]. Let $E$ be a connected nonsingular real elliptic curve diffeomorphic to $S^{1}$ and regard $a_{i}$ as a smooth map $a_{i}: M \rightarrow E$. Note that $E_{\mathbb{C}}-E$ is connected and $E$ has also totally algebraic homology. By a result of Akbulut and King (Lemma 2.7.1 and Theorem 2.8.4 of [AK2]) there exist an algebraic model $X$ of the smooth manifold $M$ and an entire rational map

$$
\Phi: X \longrightarrow E^{n_{1}} \times\left(\mathbb{C P}^{N}\right)^{n_{2}} \times\left(\mathbb{H}^{N}\right)^{n_{4}}
$$

homotopic to the product map

$$
\left(a_{1}, \ldots, a_{n_{1}}, b_{1}, \ldots, b_{n_{2}}, c_{1}, \ldots, c_{n_{4}}\right): X \longrightarrow E^{n_{1}} \times\left(\mathbb{C P}^{N}\right)^{n_{2}} \times\left(\mathbb{H}_{\mathbb{P}^{N}}\right)^{n_{4}} .
$$


Now Example 3.1 finishes the proof of the first part.

For the second part, note that the reduced $K$-group $\widetilde{K}_{0}(M)$ of complex vector bundles of any closed manifold $M$ is finitely generated. So, as above, we choose generators for $\widetilde{K}_{0}(M)$ and represent all of them by a smooth map from $M$ into a product of Grassmann varieties. Consider all these maps together with the maps $a_{i}: M \rightarrow E$, representing a generating set for the first integer cohomology group. As above, $M$ has an algebraic model $X$, so all these maps are represented by entire rational maps. In particular, any smooth complex vector bundle over $X$ is strongly algebraic. It follows that $\operatorname{Im} H^{1}(X, \mathbb{Q})=H^{1}(X, \mathbb{Q})$ and $\widetilde{K}_{0}(X) \simeq \widetilde{K}_{0}(\mathcal{R}(X, \mathbb{C}))$ and thus

$$
\widetilde{K}_{0}(X) \otimes \mathbb{Q} \simeq \widetilde{K}_{0}(\mathcal{R}(X, \mathbb{C})) \otimes \mathbb{Q},
$$

the latter being the reduced $K$-group of strongly algebraic complex vector bundles over $X$ tensored with $\mathbb{Q}$, which is known to be isomorphic to $H_{\mathbb{C}-\text { alg }}^{\text {even }}(X, \mathbb{Q}) \subset \operatorname{Im} H^{\text {even }}(X, \mathbb{Q})$ (see $\left.[\mathbf{B B K}]\right)$. Finally, it is well-known that the Chern character gives an isomorphism $\mathrm{Ch}: \widetilde{K}_{0}(\mathcal{R}(X)) \otimes \mathbb{Q} \rightarrow$ $H^{\text {even }}(X, \mathbb{Q})$ (Theorem 3.27 , p. 283 of $[\mathbf{K a}]$ ). Hence,

$$
H^{\text {even }}(X, \mathbb{Q})=H_{\mathbb{C}-\text { alg }}^{\text {even }}(X, \mathbb{Q}) \subseteq \operatorname{Im} H^{\text {even }}(X, \mathbb{Q}) \subseteq H^{\text {even }}(X, \mathbb{Q})
$$

and the proof is finished.

Theorem 3.2 does not hold for arbitrary coefficients, in particular for the cyclic group $\mathbb{Z}_{p}$, if we further require that the algebraic model $X$ is defined over rationals: Let $M$ be a closed manifold having a class $a \in H^{3}\left(M, \mathbb{Z}_{p}\right)$ with $\beta \circ P^{1}(a) \neq 0$, where $P^{1}: H^{3}\left(M, \mathbb{Z}_{p}\right) \rightarrow H^{2 p+1}\left(M, \mathbb{Z}_{p}\right)$ is the first Steenrod power for the prime $p$ and $\beta: H^{2 p+1}\left(M, \mathbb{Z}_{p}\right) \rightarrow H^{2 p+2}\left(M, \mathbb{Z}_{p}\right)$ is the Bockstein homomorphism corresponding to the exact sequence $0 \rightarrow$ $\mathbb{Z}_{p} \rightarrow \mathbb{Z}_{p^{2}} \rightarrow \mathbb{Z}_{p} \rightarrow 0$. One can take $p=3$ and $M=S^{3} / \mathbb{Z}_{3} \times S^{7} / \mathbb{Z}_{3}$, a product of lens spaces $[\mathbf{B H K}]$. In this case the triple Massey product $\langle a, a, a\rangle$ is defined and $\beta \circ P^{1}(a) \in\langle a, a, a\rangle$ (see [Mc, p. 293] or $[\mathbf{K r}]$ ). So the triple Massey product $\langle a, a, a\rangle$ is not trivial. Now suppose that $M$ has an algebraic model $X$ defined over $\mathbb{Q}$ such that

$$
\operatorname{Im} H^{3}\left(X, \mathbb{Z}_{3}\right)=H^{3}\left(X, \mathbb{Z}_{3}\right) .
$$

It follows from the naturality of Steenrod operations and Bockstein homomorphisms that for any cohomology class $b \in H^{3}\left(X_{\mathbb{C}}, \mathbb{Z}_{3}\right)$ with $i^{*}(b)=a$, $\beta \circ P^{1}(b) \neq 0$. As above the triple Massey triple product $\langle b, b, b\rangle$ is defined $[\mathbf{K r}]$ and hence is nonzero, where $i: X \rightarrow X_{\mathbb{C}}$ is any smooth projective complexification. It is well-known that for any finite abelian group $G$ the singular cohomology of $X_{\mathbb{C}}$ is isomorphic to the étale cohomology of $X$; i.e., $H^{i}\left(X_{\mathbb{C}}, G\right) \cong H^{i}\left(\left(X_{\mathbb{C}}\right)_{\text {et }}, G\right)$ (Theorem 3.12 , p. 117 in $\left.[\mathrm{Mi}]\right)$. Since $X$ is defined over rationals, $X$ is also defined over integers. Choose a big prime $q$ such that the $\bmod q$ reduction $X_{q}$ of $X$ is a nonsingular variety in some algebraically closed field of characteristic $q$. Now by [Mi, Corollary 4.2, p. 230] 
we have $H^{i}\left(\left(X_{\mathbb{C}}\right)_{\text {et }}, \mathbb{Z}_{d}\right) \cong H^{i}\left(\left(X_{q}\right)_{\text {et }}, \mathbb{Z}_{d}\right)$, for any positive integer $d$ prime to $q$. We may take $d=3$, in which case the cohomology algebra $H^{i}\left(\left(X_{q}\right)_{\text {et }}, \mathbb{Z}_{3}\right)$ supports a nontrivial Massey triple product. However, Deligne's proof of the Weil conjectures implies that any Massey higher product in $H^{i}\left(\left(X_{q}\right)_{\text {et }}, \mathbb{Z}_{3}\right)$ is trivial, as mentioned in the introduction of [DGMS]; thus we obtain a contradiction.

Indeed the same works for higher $\bmod p$ Steenrod powers. Let $M$ be a closed smooth manifold with a cohomology class $a \in H^{2 i+1}\left(M, \mathbb{Z}_{p}\right)$ satisfying $\beta \circ P^{i}(a) \neq 0$, where $P^{i}: H^{2 i+1}\left(M, \mathbb{Z}_{p}\right) \rightarrow H^{2 i p+1}\left(M, \mathbb{Z}_{p}\right)$ is the $i$-th $\bmod p$ Steenrod power and $\beta: H^{2 i p+1}\left(M, \mathbb{Z}_{p}\right) \rightarrow H^{2 i p+2}\left(M, \mathbb{Z}_{p}\right)$ is the Bockstein map. Then as above $\beta \circ P^{i}(a) \in\langle a, \ldots, a\rangle$. In particular, we have proved:

Theorem 3.4. Let $X$ be an algebraic model for the smooth manifold $M$ in the above paragraph. If $X$ is defined over $\mathbb{Q}$, then $\operatorname{Im} H^{2 i+1}\left(X, \mathbb{Z}_{p}\right) \neq$ $H^{2 i+1}\left(X, \mathbb{Z}_{p}\right)$.

Remark 3.5. Studying the example provided by $[\mathbf{B H K}]$ one can see that the class $a \in H^{3}\left(M, \mathbb{Z}_{3}\right)$ is obtained from the generators of $H^{1}\left(S^{3} / \mathbb{Z}_{3}, \mathbb{Z}_{3}\right)$ and $H^{1}\left(S^{7} / \mathbb{Z}_{3}, \mathbb{Z}_{3}\right)$ via some cohomology operations. Hence, by the results above, at least one of these lens spaces do not admit an algebraic model $X$ defined over rationals with $\operatorname{Im} H^{1}\left(X, \mathbb{Z}_{3}\right)=H^{1}\left(X, \mathbb{Z}_{3}\right)$.

We now give a contrasting result: Fix an inclusion-preserving smooth free action of a cyclic odd order group, $\mathbb{Z}_{r}$, on the telescoping sequence of odd-dimensional spheres $S^{3} \subseteq S^{5} \subseteq \cdots \subseteq S^{2 k+1} \subseteq \cdots$. Consider the corresponding telescoping sequence lens spaces $S^{3} / \mathbb{Z}_{r} \subseteq S^{5} / \mathbb{Z}_{r} \subseteq \cdots \subseteq$ $S^{2 k+1} / \mathbb{Z}_{r} \subseteq \cdots$.

Proposition 3.6. For any algebraic model $X$ of first lens space $S^{3} / \mathbb{Z}_{r}$ in the above sequence and each $k>1$, the lens space $S^{2 k+1} / \mathbb{Z}_{r}$ has an algebraic model $Y$ that contains $X$ as a subvariety. If $\operatorname{Im} H^{1}\left(Y, \mathbb{Z}_{r}\right)=H^{1}\left(Y, \mathbb{Z}_{r}\right)$ then $\operatorname{Im} H^{1}\left(X, \mathbb{Z}_{r}\right)=H^{1}\left(X, \mathbb{Z}_{r}\right)$.

Proof. Since $r$ is odd, $H^{i}\left(S^{3} / \mathbb{Z}_{r}, \mathbb{Z}_{2}\right)=0$ for $i=1,2$. Now Lemma 1.2 of [BK1] implies that any continuous vector bundle over $X$ is stably trivial. Hence, any continuous vector bundle over $X$ is strongly algebraic (see [BBK]). Consider $X$ as a submanifold of $S^{2 k+1} / \mathbb{Z}_{r}$ in the obvious way. Using a theorem of Akbulut and King (Theorem 2.8.4 of [AK2]), we can find an algebraic model $Y$ for $S^{2 k+1} / \mathbb{Z}_{r}$ having $X$ as a subvariety. In other words, the pair $X \subseteq Y$ is an algebraic model for the pair $S^{3} / \mathbb{Z}_{r} \subseteq S^{2 k+1} / \mathbb{Z}_{r}$. The rest follows easily since the $\operatorname{Im} H^{*}$ is functorial.

We believe that $S^{2 k+1} / \mathbb{Z}_{r}$, for any $k \geq 1$, has no algebraic model $X$ with $\operatorname{Im} H^{1}\left(X, \mathbb{Z}_{r}\right)=H^{1}\left(X, \mathbb{Z}_{r}\right)$. 


\section{References}

[AK1] S. Akbulut and H. King, The topology of real algebraic sets with isolated singularities, Ann. of Math., 113 (1981), 425-446, MR 0621011 (83b:58003), Zbl 0494.57004.

[AK2] S. Akbulut and H. King, Topology of Real Algebraic Sets, Mathematical Sciences Research Institute Publications, 25, Springer-Verlag, New York, 1992, MR 1225577 (94m:57001), Zbl 0808.14045.

[AK3] S. Akbulut and H. King, On approximating submanifolds by algebraic sets and a solution to the Nash conjecture, Invent. Math., 107 (1992), 87-98, MR 1135465 (93d:57051), Zbl 0781.14038.

[AK4] S. Akbulut and H. King, Algebraicity of immersions in $\mathbb{R}^{n}$,, Topology, 31 (1992), 701-712, MR 1191374 (94d:57055), Zbl 0788.57019.

[AK5] S. Akbulut and H. King, Transcendental submanifolds of $\mathbb{R}^{n}$, Comment. Math. Helv., 68 (1993), 308-318, MR 1214234 (94j:57032), Zbl 0806.57017.

[ABCKT] J. Amorós, M. Burger, K. Corlette, D. Kotschick and D. Toledo, Fundamental Groups of Compact Kähler Manifolds, Mathematical Surveys and Monographs, 44, American Mathematical Society, Providence, RI, 1996, MR 1379330 (97d:32037), Zbl 0849.32006.

[BM] E. Bierstone and P. Milman, Canonical desingularization in characteristic zero by blowing up the maximal strata of a local invariant, Invent. Math., 128 (1997), 207-302, MR 1440306 (98e:14010), Zbl 0896.14006.

[BBK] J. Bochnak, M. Buchner and W. Kucharz, Vector bundles over real algebraic varieties, K-Theory, 3 (1989), 271-289, MR 1040403 (91b:14075), Zbl 0761.14020; Erratum, K-Theory, 4 (1990), 103, MR 1076527 (91b:14075).

[BCR] J. Bochnak, M. Coste and M.-F. Roy, Real Algebraic Geometry, Ergebnisse der Mathematik und ihrer Grenzgebiete, 36, Springer, Berlin, 1998, MR 1659509 (2000a:14067), Zbl 0912.14023.

[BK1] J. Bochnak and W. Kucharz, K-theory of real algebraic surfaces and threefolds, Math. Proc. Camb. Phil. Soc., 106 (1989), 471-480, MR 1010372 (90k:14018), Zbl 0707.14006.

[BK2] J. Bochnak and W. Kucharz, Complexification of real algebraic varieties and vanishing of homology classes, Bull. London Math. Soc., 33 (2001), 32-40, MR 1798573 (2001j:14078).

[BHK] C. Bohr, B. Hanke and D. Kotschick, Cycles, submanifolds, and structures on normal bundles, preprint, math.GT/0011178.

[DGMS] P. Deligne, P. Griffiths, J. Morgan and D. Sullivan, Real homotopy theory of Kähler manifolds, Invent. Math., 29 (1975), 245-274, MR 0382702 (52 \#3584), Zbl 0312.55011.

[DM] K.H. Dovermann and M. Masuda, Uniqueness questions in real algebraic transformation groups, preprint.

[GM] P. Griffiths and J. Morgan, Rational Homotopy Theory and Differential Forms, Progress in Mathematics, 16, Birkhäuser, Boston, 1981, MR 0641551 (82m:55014), Zbl 0474.55001.

[Hi] H. Hironaka, Resolution of singularities of an algebraic variety over a field of characteristic zero (I and II), Ann. of Math., 79 (1964), 109-326, MR 0199184 (33 \#7333), Zbl 0122.38603. 
[Hu] J. Huisman, The underlying real algebraic structure of complex elliptic curves, Math. Ann., 294 (1992), 19-35, MR 1180447 (93i:14029), Zbl 0734.14019.

[Ka] M. Karoubi, K-Theory. An Introduction, Grundlehren der Mathematischen Wissenschaften, 226, Springer, Berlin, 1978, MR 0488029 (58 \#7605), Zbl 0382.55002.

[Kr] D. Kraines, Massey higher products, Trans. Amer. Math. Soc., 124 (1966), 431-449, MR 0202136 (34 \#2010), Zbl 0146.19201.

[Ku] R.S. Kulkarni, On complexifications of differentiable manifolds, Invent. Math., 44 (1978), 49-64, MR 0460731 (57 \#724), Zbl 0375.32007.

[Mc] J. McCleary, User's Guide to Spectral Sequences, Mathematics Lecture Series, 12, Publish or Perish, Wilmington, DE, 1985, MR 0820463 (87f:55014), Zbl 0577.55001.

[Mi] J.S. Milne, Étale Cohomology, Princeton Mathematical Series, 33, Princeton University Press, Princeton, New Jersey, 1980, MR 0559531 (81j:14002), Zbl 0433.14012.

[N] J. Nash, Real algebraic manifolds, Ann. of Math., 56 (1952) 405-421, MR 0050928 (14,403b), Zbl 0048.38501.

[O1] Y. Ozan, On algebraic K-theory of real algebraic varieties with circle action, J. Pure Appl. Algebra, 170(2-3) (2002), 287-293, MR 1904848 (2003e:14051).

[O2] Y. Ozan, On homology of real algebraic varieties, Proc. Amer. Math. Soc., 129 (2001), 3167-3175, MR 1844989 (2002m:14048), Zbl 1024.14025.

[S] H. Seifert, Algebraische Approximation von Mannigfaltigkeiten, Math. Zeit., 41 (1936), 1-17, Zbl 0013.36902.

[SVP] D. Sullivan and M. Vigué-Poirrier, The homology theory of the closed geodesics problem, J. Differential Geom., 11 (1976), 633-644, MR 0455028 (56 \#13269), Zbl 0361.53058.

[T] A. Tognoli, Su una congettura di Nash, Ann. Scuola Norm. Sup. Pisa, 27 (1973), 167-185, MR 0396571 (53 \#434), Zbl 0263.57011.

Received April 9, 2002.

\author{
Mathematics Department \\ Middle East Technical University \\ 06531 ANKARA \\ TURKEY \\ E-mail address: ozan@metu.edu.tr
}

